\title{
Identification of a three-gene-based prognostic model in multiple myeloma using bioinformatics analysis
}

\author{
Ying Pan ${ }^{\text {Equal first author, } 1}$, Ye Meng ${ }^{\text {Equal first author, } 1}$, Zhimin Zhai ${ }^{\text {Corresp., } 1}$, Shudao Xiong ${ }^{\text {Corresp. } 1}$ \\ ${ }^{1}$ Department of Hematology, The Second Affiliated Hospital of Anhui Medical University, Hefei, China \\ Corresponding Authors: Zhimin Zhai, Shudao Xiong \\ Email address: zhaizhimin889@163.com, 594423220@qq.com
}

Background: Multiple myeloma (MM), the second most hematological malignancy, has high incidence and remains incurable till now. The pathogenesis of MM is poorly understood. This study aimed to identify novel prognostic model for MM on gene expression profiles.

Methods: Gene expression datas of MM (GSE6477, GSE136337) were downloaded from Gene Expression Omnibus (GEO) database. The differentially expressed genes (DEGs) in GSE6477 between case samples and normal control samples were screened by the limma package. Meanwhile, enrichment analysis was conducted, and a protein-protein interaction (PPI) network of these DEGs was established by STRING and cytoscape software. Co-expression modules of genes were built by Weighted Correlation Network Analysis (WGCNA). Key genes were identified both from hub genes and the DEGs. Univariate and multivariate Cox congression were performed to screen independent prognostic genes to construct a predictive model. The predictive power of the model was evaluated by Kaplan-Meier curve and timedependent receiver operating characteristic (ROC) curves. Finally, univariate and multivariate Cox regression analyse were used to investigate whether the prognostic model could be independent of other clinical parameters.

Results:GSE6477, including 101 case and 15 normal control, were screened as the datasets.178 DEGs were identified, including 59 up-regulated and 119 down-regulated genes. In WGCNA analysis, module black and module purple were the most relevant modules with cancer traits, and 92 hub genes in these two modules were selected for further analysis. Next, 47 genes were chosen both from the DEGs and hub genes as key genes. Three genes (LYVE1, RNASE1, and RNASE2) were finally screened by univariate and multivariate Cox regression analyses and used to construct a risk model. In addition, the three-gene prognostic model revealed independent and accurate prognostic capacity in relation to other clinical parameters for MM patients.

Conclusion:In summary, we identified and constructed a three-gene-based prognostic model that could beused to predict overall survival of MM patients. 
1 Identification of a three-gene-based prognostic model in Multiple Myeloma using

2 bioinformatics analysis

3

4 Ying Pan , *1 Ye Meng , *1 Zhimin Zhai ,1 and Shudao Xiong1

5

61 Department of Hematology, The Second Affiliated Hospital of Anhui Medical University, Hefei, Anhui

7 230601, China.

8

$9 *$ Contributed equally

10

Correspondence should be addressed to Zhimin Zhai; Email: zzzm889@163.com

12

Shudao Xiong; Email: xshdao@ahmu.edu.cn

ABSTRACT

Background: Multiple myeloma (MM), the second most hematological malignancy, has high incidence and remains incurable till now. The pathogenesis of $\mathrm{MM}$ is poorly understood. This study aimed to identify a novel prognostic model for MM on gene expression profiles.

Methods: Gene expression datas of MM (GSE6477, GSE136337) were downloaded from Gene Expression Omnibus (GEO) database. The differentially expressed genes (DEGs) in GSE6477 between case samples and normal control samples were screened by the limma package. Meanwhile, enrichment analysis was conducted, and a protein-proteininteraction (PPI) network of these DEGs was established by STRING and cytoscape software.Co-expression modules of genes were built by Weighted Correlation Network 
Cox congression were performed to screen independent prognostic genes to construct a predictive model. The predictive power of the model was evaluated by Kaplan-Meier curve and time-dependent receiver operating characteristic (ROC) curves. Finally, univariate and multivariate Cox regression analyse were used to investigate whether the prognostic model could be independent of other clinical parameters.

Results: GSE6477, including 101 case and 15 normal control, were screened as the datasets. 178DEGs were identified, including 59 up-regulated and 119 down-regulated genes. In WGCNA analysis, module black and module purple were the most relevant modules with cancer traits, and 92 hub genes in these two modules were selected for further analysis. Next, 47 genes were chosen both from the DEGs and hub genes as key genes. Three genes (LYVE1, RNASE1, and RNASE2) were finally screened by univariate and multivariate Cox regression analyses and used to construct a risk model. In addition, the three-gene prognostic model revealed independent and accurate prognostic capacity in relation to other clinical parameters for MM patients.

Conclusion: In summary, we identified and constructed a three-gene-based prognostic model that could be used to predict overall survival of MM patients.

Keywords: Multiple myeloma, prognosis, WGCNA, bioinformatics analysis, prognostic model

\section{INTRODUCTION}

Multiple myeloma ( $\mathrm{MM}$ ) is the second most common hematological malignancy, and caused by abnormal monoclonal plasma cells. It accounts for $10 \%$ of all hematological malignancies (Mateos MV, 2017). Many novel therapy strategies have been developed, and the survival of MM patients has improved considerably(Nooka AK, 2019; Ocio EM, 2014). However, the prognosis remains poor and the disease is still incurable (Nijhof IS, 2018). It is of great significance to identify novel cancer-specific prognostic biomarkers and construct prognostic model to find high-risk MM patients.

The prognostic models of MM usually include clinical information and cytogenetic abnormalities (QiT, 2020). 
International Staging System (ISS) and the revised ISS (R-ISS) are the most commonly used prognostic models. Recently, some prognostic models based on gene expression profile have been used to predict prognosis in MM, and even better than R-ISS (Liu Y, 2019; Bai H, 2020). Besides, new technologies and methods such as single cell RNA-Seq (scRNA-Seq) have been used to predict the prognosis of MM (Jang JS, 2019). Weighted gene co-expression network (WGCNA) is also a novel method which uses numerous data in public databases to explore the underlying mechanism of gene networks, and usually used for gene prediction in large-scale datasets in recent years (Zhang B, 2005; Langfelder P, 2008).It first divides gene co-expression networks of complicated biological processes into several modules, then analyze their potential association with clinical traits to look for key modules and hub genes (Zhang B, 2005). It has been widely utilized in many malignant diseases as well as non-malignant diseases (Li W, 2020; Xu X, 2020). However, it is rarely used in MM.

In the present study, we aimed to use WGCNA method to identify co-expression networks associated with $\mathrm{MM}$ and to find potential prognostic biomarkers and construct prognostic model for MM patients on gene expression profiles.

\section{MATERIALS AND METHODS}

\section{Data Preparation}

The raw data of GSE6477 and GSE136337 were downloaded from the gene expression omnibus (GEO) website (http://www.ncbi.nlm.nlh.gov/geo/). There were 101 case and 15 normal control in GSE6477, was based on GPL96Platforms ([HG-U133A] Affymetrix Human Genome U133A Array).There were 401 cases with overall survival (OS) and other clinical information was chosen to identify prognostic genes and construct the prognostic model in GSE136337 Dataset GSE136337. All the patients were randomly divided into 2 groups (60\% as the training set and $40 \%$ as the test set). The Robust multi-arrayaverage (RMA) method was used to normalize data.

\section{Analysis of differentially Expressed Genes (DEGs)}

Limma package was used to identify differentially expressed genes (DEGs) (Ritchie ME, 2015), and the threshod value was |log2foldchange $\mid>1.0$ and adj.P.Val $<0.05$.

\section{GO term and KEGG pathway enrichment analysis}


86

To better explore the biological significance of DEGs, Gene Ontology (GO) and Kyoto Encyclopedia of Genes and Genomes (KEGG) pathway enrichment analysis were performed (YuG, 2012). qvalue $<0.05$ was used as the cutoff value.

\section{A protein-protein interaction (PPI) network}

A PPI network of these DEGs was constructed by using the Search Tool for the Retrieval of Interacting Genes (STRING) database (https://string-db.org/) (Szklarczyk D, 2015).

\section{Co-expression network analysis}

The co-expression network was constructed by WGCNA to explore the correlation of genes (Top50\%mad) and search for important interacted gene modules. PickSoftThreshold function of the WGCNA was used to calculate the soft thresholding power $\beta$ value, and the $\beta$ value was set as 6 . Then, we constructed the coexpression modules using WGCNA algorithms with R software package. Next, Gene significance (GS) and Module membership measures were used to identify genes with high clinical significance as well as high Module membership. Module membership was the correlation between gene expression profile and module eigengene. While, GS was the absolute value of the correlation between gene expression and module traits.

\section{Identification of prgnostic key genes and construction of the prognostic model}

Key genes were identified both from hub genes and the DEGs (Xia L, 2018). All the patients were randomly divided into 2 groups ( $60 \%$ as the training set and $40 \%$ as the test set) and univariate and multivariate Cox regression analyses were used to investigate the correlation between OS and the expression level of key genes. P-value $<0.05$ was considered of significant difference in the univariate analysis. Key genes with $\mathrm{P}<$ 0.05 based on the univariate analysis were further included in the multivariate Cox regression analysis. Finally, a prognostic gene signature of MM patients was established based on a linear combination of the regression coefficient derived from the multivariate Cox regression model $(\beta)$ multiplied with its expression level. The patients were separated into low- and high-risk groups based on the optimal cut-off value (Camp RL, 2004) Kaplan-Meier (K-M) survival curves, time-dependent receiver operating characteristic (ROC) curve analyses and area under the curve (AUC) were used to evaluate the prediction accuracy of our model (Heagerty PJ, 2005). We also validated the prognostic model in the test set and whole set by K-M curves, ROC curve analyses, and AUC. 


\section{Identification of Independent Prognostic Parameters of MM}

To investigate independent prognostic parameters and to identify whether the prognostic model

was the independent prognostic parameter, univariate and multivariate Cox regression analyses were performed using the Cox regression model method with forwarding stepwise procedure. Other clinical parameters including age, gender, number of transplantation (NOT), tumor staging (ISS and R-ISS), cytogenetic abnormalities, lactate dehydrogenase (LDH) level, albumin level, $\beta 2$-microglobulin level, and percentage of abnormal plasma cell in bone marrow. P-value $<0.05$ was considered of significant difference. Also, we compared the AUC of our model with other conventional models such as age, gender, ISS, and R-ISS models.

\section{RESULTS}

\section{Identification of DEGs in MM and the enrichment of these DEGs}

The DEGs of GSE6477 were screened by the limma package. A total of 178 DEGs were identified, including 59 upregulated genes and 119 downregulated genes in MM case samples compared to normal control samples

(Fig.1a). The DEGs were shown on a heatmap (Fig.1b). GO analysis showed that these genes were significantly enriched in neutrophil activation, humoral immune response, and atructural constituent of ribosome (Fig.1c). In KEGG analysis, the DEGs were mainly enriched in Asthma, Staphylococcus aureus infection, and Ribosome (Fig.1d).Next, A PPI network, as demonstrated in Fig.2, was constructed using the online STRING website to understand the interaction among the related DEGs in MM.

\section{Co-expression analysis and hub genes identification.}

WGCNA was used to construct co-expression network and to find the modules of highly correlated genes. Firstly, we calculated the soft thresholding power $\beta$, and the power of $\beta$ was set at 6 to ensure a scale-free network in our research(Fig. 3a-d). Next, we identified 15 modules for further analysis (Fig.4a). The heatmap and meta-modules were used to visualize the gene network (Fig. 4b, c). Among these 15 modules, module black and module purple were the most relevant modules with cancer traits (Fig.4d). Subsequently, intramodular analysis of the genes in the 15 modules was followed. And genes in the black and purple modules were found to have high correlations with MM (Fig.4e,f). Finally, genes in these two modules were selected as hub genes according to the cut-off criteria: module membership values $>0.80$ and GS values $>0.2$. 
Totally, there were 92 hub genes from black and purple modules that were chosen for next analysis.

\section{Identification of survival related key genes and construction of three-gene prognostic signature}

Key genes were identified both from hub genes and the DEGs. Totally, 47 key genes were identified (Fig.5). To evaluate the prognostic values of these key genes in MM patients, we next performed a univariate Cox regression, and identified 8 genes significantly related to OS in MM patients. Then, a stepwise multivariate Cox regression analysis was performed, and three genes (LYVE1, RNASE1, and RNASE2) were eventually selected to construct a prognostic model (Fig.6). Risk score $=(-0.59714 *$ expression level of LYVE1) $+(-$ 1.35971* expression level of RNASE1 $)+(0.28240$ * expression level of RNASE2). Next, we calculated the optimal cut-off value for the three-gene expression risk score with X-Tile software, and divided patients in training set $(n=241)$ into high risk group and low risk group. The K-M curve showed that high risk group had poorer prognosis than low risk group $(P<0.0001)$ (Fig.7). The AUCs of time-dependent ROC curves were calculated to assess the prognostic capacity of the three-gene signature. The AUCs of risk scores in training set at 8-,10-, and 12-year survival times were $0.717,0.720$ and 0.800 (Fig.8a).

To verify the predictive value of the three-gene signature, we used the internal test set $(n=160)$ and whole set $(n=401)$ to assess the results from the training set. Consistent of the findings of the training set,the K-M survival curves of test set and whole set revealed that worse OS existed in the high risk groups (Fig.7). The AUCs of risk scores in test set at 8-,10-, and 12-year survival times were 0.709, 0.768 and 0.766 (Fig.8b), and the whole set were $0.714,0.741$ and 0.783 ,respectively (Fig.8c). We also compared the prognostic capacity of our model with other conventional models, including age, gender, ISS, and R-ISS stage in whole set. The AUCs for 8-, 10-, and 12-year OS predictions for age were $0.500,0.518$, and 0.478 ; and for gender were 0.516 , 0.506 , and 0.450 (Fig.8d-f). The AUCs of ISS stage at 8-, 10-, and 12-year survival times were $0.503,0.547$, and 0.633; and of R-ISS stage were $0.613,0.616$, and 0.634 (Fig.8d-f). Our model had the highest AUCs, indicating a superior prognostic value. Together, these results demonstrated that our model was capable of predicting OS of MM patients.

\section{The prognostic model is independent for MM patients.}

The univariate and multivariate Cox regression analyses were utilized to investigate independent prognostic parameters and identify whether the three-gene prognostic model was the independent prognostic parameter in $401 \mathrm{MM}$ patients with complete clinical information from GSE16337. Univariate Cox regression 
177

178

179

180

181

182

183

184

185

186

187

188

189

190

191

192

193

194

195

196

197

198

199

200

201

202

203

204

205

206

207

analysis showed that NOT, tumor stage (ISS and R-ISS), $\mathrm{t}(11,14)$, albumin level, $\beta 2$-microglobulin level, and the prognostic model had prognostic values (Fig.9a). After the multivariate Cox regression analysis, NOT, RISS, $t(11,14)$, LDH level and the prognostic model were proved to be independent prognostic factors for OS of MM patients (Fig.9b).

\section{Correlation between the prognostic model with clinical characters}

Correlation between risk score and various clinical parameters were shown in Fig.10. Our results indicated that risk score was significantly correlated with ISS, and R-ISS stage; patients in phase III of ISS or R-ISS had the highest risk score when compared with patients in phase I and II (Fig.10). However, no differences were found between risk score and age or gender or NTO (Table.1). Besides, the relationships between the three genes in the prognostic model with clinical characters were also analyzed. We found that as LYVE1 expression, which was higher in males than in females, decreased, ISS stage in MM patients increased significantly (Fig.10a, Table.1). Additionally, as LYVE1 and RNASE1 expression decreased, R-ISS stage in MM patients increased (Fig.10b,c). These findings showed that LYVE1 and RNASE1 exerted protective effects against disease progression.

\section{DISCUSSION}

WGCNA is an important and helpful method which describes the pattern of gene association between different samples, and usually used for gene prediction in large-scale datasets (Zhang B,2005; Langfelder P, 2008). The main advantage of this method is that it can cluster genes into co-expression modules, as well as build a bridge between gene expression changes and sample characteristics. Thus, WGCNA has been widely used in various malignant and non-malignant diseases (Chen J, 2020; Chen X, 2019; Chen X, 2018; Li Z, 2020; Zheng JN, 2020). In cancer research, more and more researchers have applied WGCNA for developing new prognostic biomarkers (Xu X, 2020; Chen J, 2020; Chen X, 2019; Chen X, 2018). In this study, we firstly used an integrated analysis from both WGCNA and DEGs to screen potential biomarkers related to prognosis of MM in GEO databases.

Totally, 178 DEGs were identified, including 59 up-regulated genes and 119 down-regulated genes in GSE6477. Next, we applied WGCNA to construct a co-expression network for evaluating the relationshipsbetween genes and modules. In WGCNA, genes were clustered into 15 modules, and the blackmodule and purple module were identified to be the most relevant modules with cancer traits.

PeerJ reviewing PDF | (2020:11:55385:1:1:NEW 18 Feb 2021) 
208

209

210

211

212

213

214

215

216

217

218

219

220

221

222

223

224

225

226

227

228

229

230

231

232

233

234

235

236

237

238

239

According to the cut-off criteria: module membership values $>0.80$ and GS values $>0.2$, a total of 92 hub genes from these two modules were selected. Key genes were excavated from both the hub genes and the DEGs. Totally, 47 key genes were identified and selected for survival analysis. Using univariate and multivariate Cox regression, we screened three independent prognostic genes and constructed a predictive model. The prognostic genes were LYVE1, RNASE1, and RNASE2. The patients were divided into high- and low-risk groups based on the risk score. Training set, test set and whole set were utilized to validate the prognostic capacity of the our model. Besides, the three-gene prognostic model revealed independence and accuracy in relation to other clinical parameters for MM patients. Our model was the first prognostic model for survival times of MM via integrative bioinformatics analysis.

All of the genes in the three-gene signature have not been previously reported to be associated with MM. LYVE1 is a hyaluronic acid receptor. Its main function is to transport hyaluronidase in extracellular matrix to lymphatic fluid. Also, it involves in the generation of lymphatic vessels and the activation of lymphocytes (Arimoto S 2018). High expression level of LYVE1 in tumor tissue can induce tumor cell proliferation, lymphangiogenesis and lymphatic metastasis, and was reported to be closely related to poor prognosis in many cancers, including lung cancer, renal cancer, and breast cancer (Li P 2018; Schraml P 2019; Hunter S 2019). However, LYVE1 might also play anti-tumor role in some cancers. In papillary thyroid carcinoma, LYVE1 was proved to be decreased and associated with poor prognosis (Wu M 2019). Latil et al. (2003) reported that LYVE1 was downregulated in prostate cancer and was related to the relapse. In hepatocellular carcinoma and ovarian cancer, its expression was also decreased and played the tumor-suppressive effect (Llovet JM 2006; Gao Y 2017). However, its expression and role in MM has not been reported. In this study, we found that the downregulation of LYVE1 in MM was associated with worse prognosis. In addition, the expression level of LYVE1 was closely related to clinical stage (ISS and R-ISS).

RNASE1, belongs to the ribonuclease (RNase) A gene superfamily, acts to regulate the vascular homeostasis of extracellular RNA (Bedenbender K 2019). In gastric cancer, RNASE1 acted as a tumor suppressor gene, as its expression was demonstrated to decrease progressively from the normal, primary cancer and metastatic cells (Wang L 2006). While in prostate cancer, overexpression of RNASE1 was associated with the poor survival (Gao L 2020).

RNASE2, another member of the RNase A gene family, mainly involves in immune function and plays an important role in toll-like receptor activation (Ostendorf T 2020). Studies have identified that combination 
240

analysis of RNASE2 with other genes has independent prognostic value in kidney renal clear cell carcinoma (Xiang Y 2020) and renal cell carcinoma (Qin X 2021). It has also been found to be upregulated in acute lymphoblastic leukemia (Niini T 2002). The upregulation of RNASE2 in gastric cancer was associated with poorer survival (Wang WS, 2020). However, its role in MM is unknown. In our study, overexpressed RNASE2 was found to be associated with worse prognosis in MM patients. The tumor suppressor effect of RNASE2 in MM and its molecular mechanisms need further study.

There are several advantages and limitations in this study. The GEO databases have enough sample size and provide a relatively believable basis for bioinformatics analysis. Besides, in this study, we used an integrated analysis from both WGCNA and DEGs to screen potential biomarkers, which may also make our results more credible. Although our results were validated in other GEO databases, it would be more reliable to validate the significance of prognostic

model in real-world clinical MM cohorts. Moreover, biological functions and underlying molecular mechanisms of these key genes in MM are still needed to be explored in the future research.

\section{CONCLUSIONS}

In summary, we used comprehensive study including WGCNA, DEGs screening, univariate and multivariate Cox regression to construct a three-gene-based prognostic model, which exhibits an

effective prognostic value for distinguishing MM patients with poor OS.

\section{ACKNOWLEDGMENTS}

Not applicable.

\section{REFERENCE}

Mateos MV, San Miguel JF. 2017. Management of multiple myeloma in the newly diagnosed patient. Hematology Am Soc Hematol Educ Program. 2017:498-507.

Nooka AK, Kaufman JL, Hofmeister CC, Joseph NS, Heffner TL, Gupta VA, Sullivan HC, Neish AS, Dhodapkar MV, Lonial S. 2019. Daratumumab in multiple myeloma. Cancer. 125:2364-2382. doi: 10.1002/cncr.32065. 
268

269

270

271

272

273

274

275

276

277

278

279

280

281

282

283

284

285

286

287

288

289

290

291

292

293

294

295

296

Ocio EM, Richardson PG, Rajkumar SV, Palumbo A, Mateos MV, Orlowski R, Kumar S, Usmani S, Roodman D, Niesvizky R, Einsele H, Anderson KC, Dimopoulos MA, Avet-Loiseau H, Mellqvist UH, Turesson I, Merlini G, Schots R, McCarthy P, Bergsagel L, Chim CS, Lahuerta JJ, Shah J, Reiman A, Mikhael J, Zweegman S, Lonial S, Comenzo R, Chng WJ, Moreau P, Sonneveld P, Ludwig H, Durie BG, Miguel JF. 2014. New drugs and novel mechanisms of action in multiple myeloma in 2013: A report from the International Myeloma Working Group (IMWG). Leukemia 28, 525-542.

Nijhof IS, van de Donk NWCJ, Zweegman S, Lokhorst HM. 2018. Current and new therapeutic strategies for relapsed and refractory multiple myeloma: an update. Drugs. 78:19-37.

Qi T, Qu J, Tu C, Lu Q, Li G, Wang J, Qu Q. 2020. Super-Enhancer Associated Five-Gene Risk Score Model Predicts Overall Survival in Multiple Myeloma Patients. Front Cell Dev Biol. 3;8:596777.

Liu Y, Yang N, Peng X, Liu G, Zhong H, Liu L. 2019. One-lincRNA and five-mRNA based signature for prognosis of multiple myeloma patients undergoing proteasome inhibitors therapy. Biomed Pharmacother. 18:109254.

Bai H, Xu P, Wang L, Chen B. A centromere-associated gene score for rapid determination of risk in multiple myeloma. Am J Transl Res. 2020 Jun 15;12(6):2425-2438.

Jang JS, Li Y, Mitra AK, Bi L, Abyzov A, van Wijnen AJ, Baughn LB, Van Ness B, Rajkumar V, Kumar S, Jen J. 2019. Molecular signatures of multiple myeloma progression through single cell RNA-Seq. Blood Cancer J. 3;9(1):2.

Zhang B, Horvath S. 2005. A general framework for weighted gene co-expression network analysis. Stat Appl Genet Mol Biol. 4:Article17. doi: 10.2202/1544-6115.1128.

Li W, Wang L, Wu Y, Yuan Z, Zhou J. 2020. Weighted gene co-expression network analysis to identify key modules and hub genes associated with atrial fibrillation. Int J Mol Med. 45(2):401-416. doi:10.3892/ijmm.2019.4416

Xu X, Xu L, Huang H, Li J, Dong S, Jin L, Ma Z, Li L. 2020. Identification of Hub Genes as Biomarkers Correlated with the Proliferation and Prognosis in Lung Cancer: A Weighted Gene Co-Expression Network Analysis. Biomed Res Int.2020:3416807.

Ritchie ME, Phipson B, Wu D, Hu Y, Law CW, Shi W, Smyth GK. 2015. limma powers differential expression analyses for RNA-sequencing and microarray studies. Nucleic Acids Res. 43(7):e47.

Yu G, Wang LG, Han Y, He QY. clusterProfiler: an R package for comparing biological themes among gene clusters. OMICS. 2012;16(5):284-287.

Szklarczyk D, Franceschini A, Wyder S, Forslund K, Heller D, Huerta-Cepas J, Simonovic M, Roth A, Santos A, 
297

298

299

300

301

302

303

304

305

306

307

308

309

310

311

312

313

314

315

316

317

318

319

320

321

322

323

324

Tsafou KP, Kuhn M, Bork P, Jensen LJ, von Mering C. 2015. “STRING v10: protein-protein interaction networks, integrated over the tree of life, ” Nucleic Acids Research, vol. 43, no. D1, pp. D447-D452.

Xia L, Su X, Shen J, Meng Q, Yan J, Zhang C, Chen Y, Wang H, Xu M. 2018. ANLN functions as a key candidate gene in cervical cancer as determined by integrated bioinformatic analysis. Cancer Manag Res. 10:663-670

Camp RL, Dolled-Filhart M, Rimm DL. 2004. X-tile: a new bio-informatics tool for biomarker assessment and outcome-based cut-point optimization. Clin Cancer Res.10:7252-9.

Heagerty PJ, Zheng Y. 2005. Survival model predictive accuracy and ROC curves. Biometrics. 61:92-105.

Chen J , Cai Y , Xu R , Pan J, Zhou J, Mei J. 2020. Identification of four hub genes as promising biomarkers to evaluate the prognosis of ovarian cancer in silico. Cancer Cell International, 20:270.

Chen X, Hu L, Wang Y, Sun W, Yang C. 2019. Single cell gene co-expression network reveals FECH/CROT signature as a prognostic marker. Cells. 8(7):698.

Chen X, Yang C, Xie S, Cheung E. 2018. Long non-coding RNA GAS5 and ZFAS1 are prognostic markers involved in translation targeted by miR-940 in prostate cancer. Oncotarget. 9(1):1048-62.

Li Z, Cui Y, Feng J, Guo Y. 2020. Identifying the pattern of immune related cells and genes in the peripheral blood of ischemic stroke. J TransI Med. 18(1):296.

Zheng J N , Li Y, Yan Y M , Shi H, Zou TT, Shao WQ, Wang Q. 2020. Identification and Validation of Key Genes Associated With Systemic Sclerosis-Related Pulmonary Hypertension. Frontiers in Genetics, 11: 816.

Arimoto S, Hasegawa T, Takeda D, Saito I, Amano R, Akashi M, Komori T. 2018. Lymphangiogenesis and lymphno demetastasis in oral squamous cell carcinoma. Anticancer Res, 38(11):6157-6162

Li P, Cong Z, Qiang Y, Xiong L, Tang L, Zhang Y, Wu H, Yi J, Jing H, Li D, Shen Y. 2018 . Clinical significance of CCBE1 expression in lung cancer. Mol Med Rep. 17:2107-12. 10.3892/mmr.2017.8187

Schraml P, Athelogou M, Hermanns T, Huss R, Moch H. 2019. Specific immune cell and lymphatic vessel signatures identified by image analysis in renal cancer. Modern Pathol. 32:1042-52.

Hunter S, Nault B, Ugwuagbo KC, Maiti S, Majumder M. 2019. Mir526b and Mir655 promote tumour associated angiogenesis and lymphangiogenesis in breast cancer. Cancers. 11(7):938.

Wu M, Yuan H, Li X, Liao Q, Liu Z. 2019. Identification of a Five-Gene Signature and Establishment of a prognostic Nomogram to Predict Progression-Free Interval of Papillary Thyroid Carcinoma. Front Endocrinol (Lausanne). 10:790.

Peer] reviewing PDF | (2020:11:55385:1:1:NEW 18 Feb 2021) 
325

326

327

328

329

330

331

332

333

334

335

336

337

338

339

340

341

342

343

344

345

346

347

348

349

350

351

352

353

354

Latil A, Bieche I, Chene L, Laurendeau I, Berthon P, Cussenot O, Vidaud M. 2003. Gene expression profiling in clinically localized prostate cancer: a four-gene expression model predicts clinical behavior. Clin Cancer Res. 9:5477-85.

Llovet JM, Chen Y, Wurmbach E, Roayaie S, Fiel MI, Schwartz M, Thung SN, Khitrov G, Zhang W, Villanueva A, Battiston C, Mazzaferro V, Bruix J, Waxman S, Friedman SL. 2006. A molecular signature to discriminate dysplastic nodules from early hepatocellular carcinoma in HCV cirrhosis. Gastroenterology. 131:1758-67.

Gao Y, Liu X, Li T, Wei L, Yang A, Lu Y, Zhang J, Li L, Wang S, Yin F. 2017. Cross-validation of genes potentially associated with overall survival and drug resistance in ovarian cancer. Oncol Rep. 37:3084-92.

Bedenbender K, Scheller N, Fischer S, Leiting S, Preissner KT, Schmeck BT, Vollmeister E. 2019. Inflammationmediated deacetylation of the ribonuclease 1 promoter via histone deacetylase 2 in endothelial cells. FASEB J. 33(8):9017-29.

Wang L, Zhu JS, Song MQ, Chen GQ, Chen JL. 2006. Comparison of gene expression profiles between primary tumor and metastatic lesions in gastric cancer patients using laser microdissection and cDNA microarray. World J Gastroenterol. 12(43):6949-54.

Gao L, Meng J, Zhang Y, Gu J, Han Z, Wang X, Gao S. 2020. Development and validation of a six-RNA binding proteins prognostic signature and candidate drugs for prostate cancer. Genomics. 112(6):4980-92.

Ostendorf T, Zillinger T, Andryka K. Schlee-Guimaraes TM, Schmitz S, Marx S, Bayrak K, Linke R, Salgert S, Wegner J, Grasser T, Bauersachs S, Soltesz L, Hübner MP, Nastaly M, Coch C, Kettwig M, Roehl I, Henneke M, Hoerauf A, Barchet W, Gärtner J, Schlee M, Hartmann G, Bartok E. 2020. Immune Sensing of Synthetic, Bacterial, and Protozoan RNA by Toll-like Receptor 8 Requires Coordinated Processing by RNase T2 and RNase2. Immunity. 52:591-605.e6.

Xiang Y, Zhou S, Hao J, Zhong C, Ma Q, Sun Z, Wei C. Development and validation of a prognostic model for kidney renal clear cell carcinoma based on RNA binding protein expression. Aging (Albany NY). 12(24):2535672.

Qin X, Liu Z, Yan K, Fang Z, Fan Y. 2021. Integral Analysis of the RNA Binding Protein-associated Prognostic Model for Renal Cell Carcinoma. Int J Med Sci. 18(4):953-963.

Niini T, Vettenranta K, Hollmén J, Larramendy ML, Aalto Y, Wikman H, Nagy B, Seppänen JK, Ferrer Salvador A, Mannila H, Saarinen-Pihkala UM, Knuutila S. 2002. Expression of myeloid-specific genes in childhood acute lymphoblastic leukemia - a cDNA array study. Leukemia. 16(11):2213-21.

Wang WS, Zhu JR, Mu ZY, Liu BY, Wang B, Chen DF, Wen ZL. 2020. Expression and clinical significance of

PeerJ reviewing PDF | (2020:11:55385:1:1:NEW 18 Feb 2021) 
355 immunogenomics in gastric adenocarcinoma. Clin J Gastroenterol Hepatol. 29; 507-514. 
Figure 1

The DEGs identified in GSE6477.

(a). Volcano map of DEGs between MM case group and normal control group. (b). Heatmap of the DEGs. (c). The top $20 \mathrm{GO}$ terms in the enrichment analysis of DEGs. (d) The top 30 KEGG pathways in the enrichment analysis of DEGs.

a

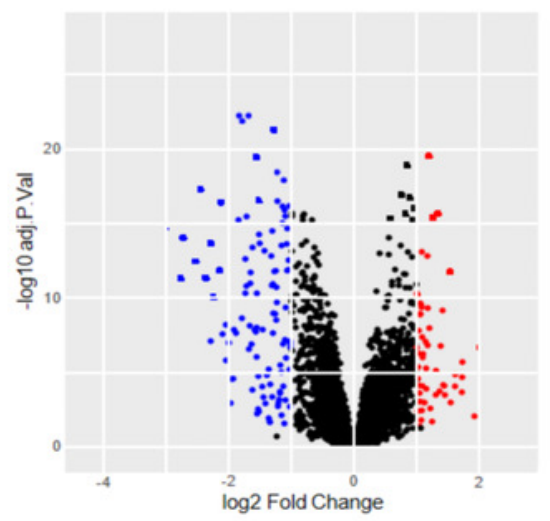

C

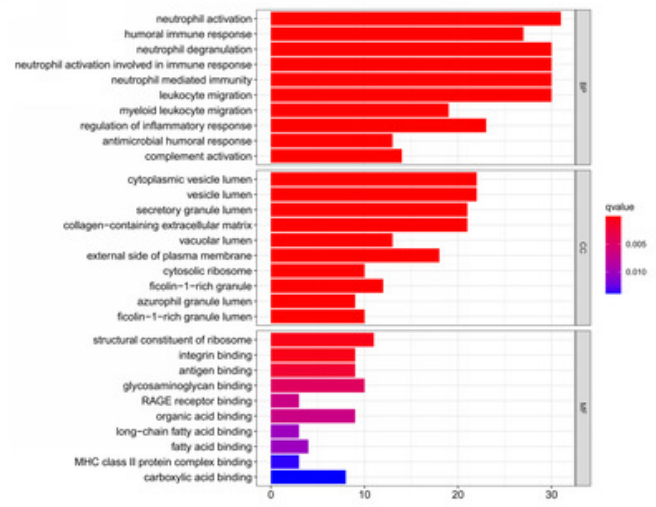

b

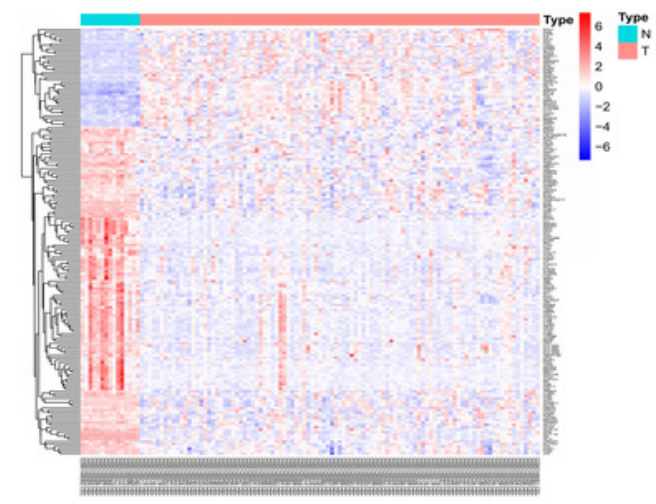

d

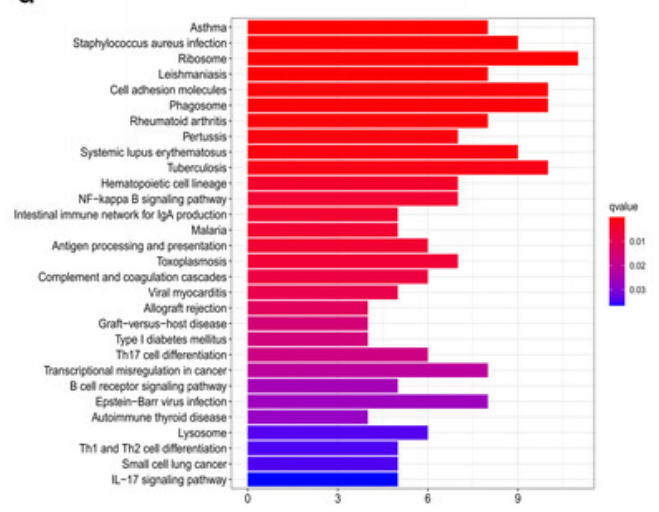




\section{Figure 2}

\section{Analysis of the PPI network.}

\section{DEGs were filtered into the DEGs PPI network complex.}

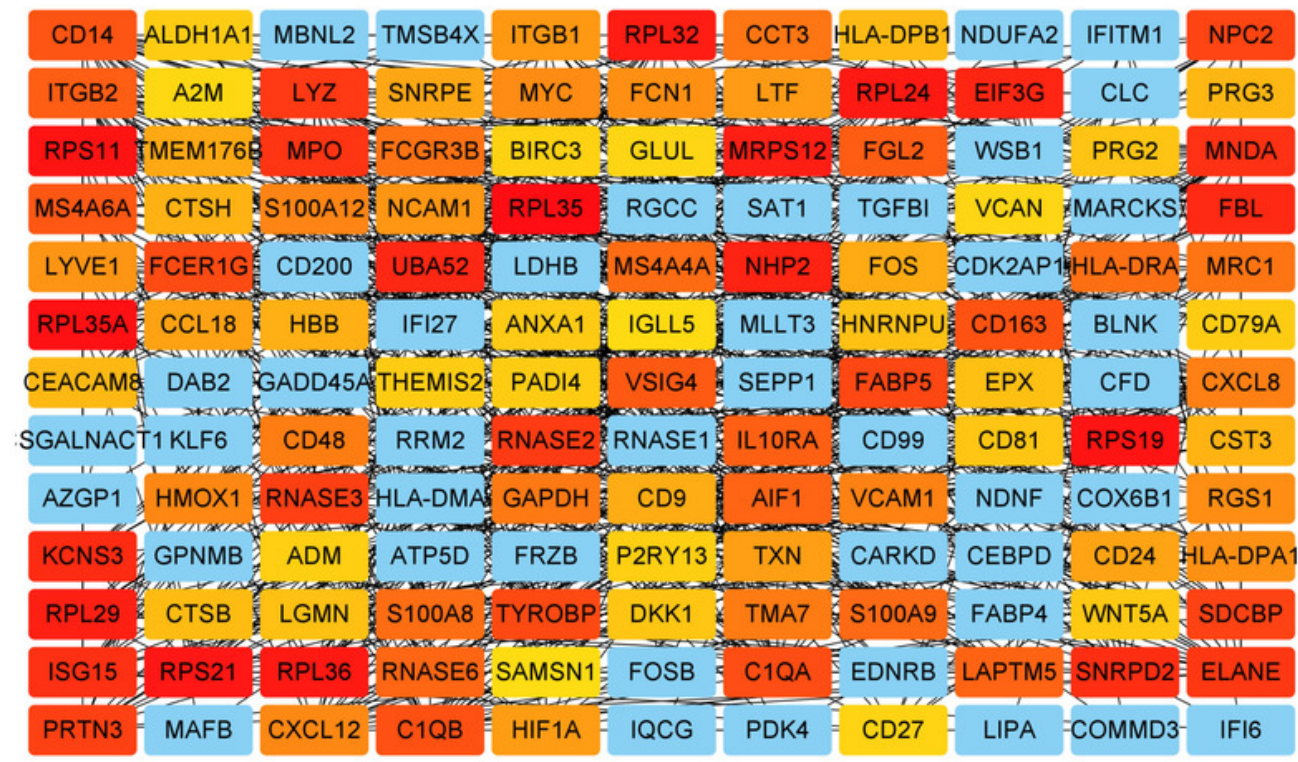


Figure 3

Determination of soft thresholding power.

(a). Analysis of the scale-free fitting indices for various soft thresholding powers. (b). Mean connectivity analysis of various soft thresholding powers. (c). Histogram of the connection distribution when $\beta=6$. $d$. Check scale-free topology when $\beta=6$.
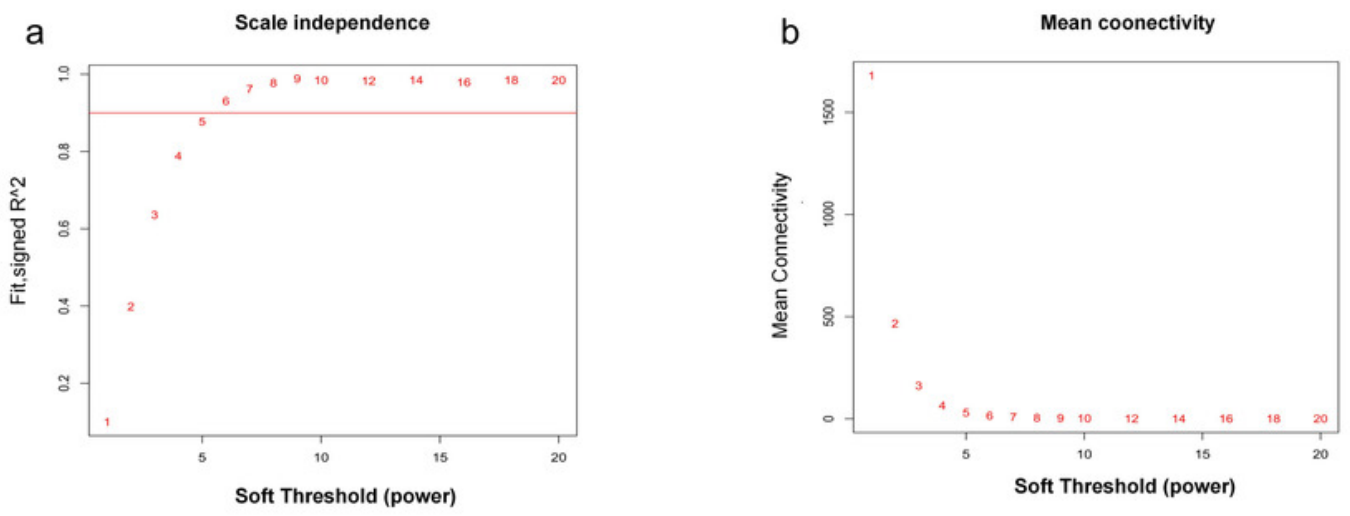

C

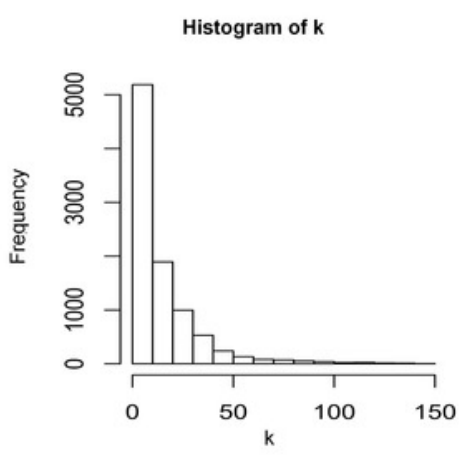

d

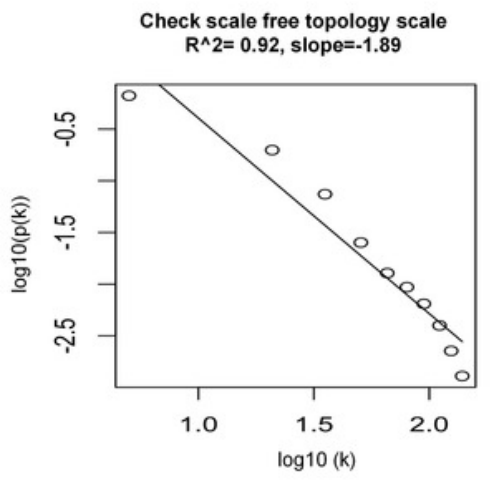




\section{Figure 4}

Identification of modules associated with MM clinical traits.

(a). Clustering dendrograms of genes based on co-expression network analysis. In total, 15 co-expression modules were constructed and shown in different colors. (b). A heatmap of all the genes. The light color indicated a low overlap, and the darker red color indicated higher overlap. (c). The eigengene dendrogram and eigengene adjacency plot. (d). Module-trait association. The purple and black gene modules were the most relevant modules with cancer traits. (e). A scatter plot of gene significance for MM versus the module membership in the black module. (f). A scatter plot of gene significance for MM versus the module membership in the purple module. 
a

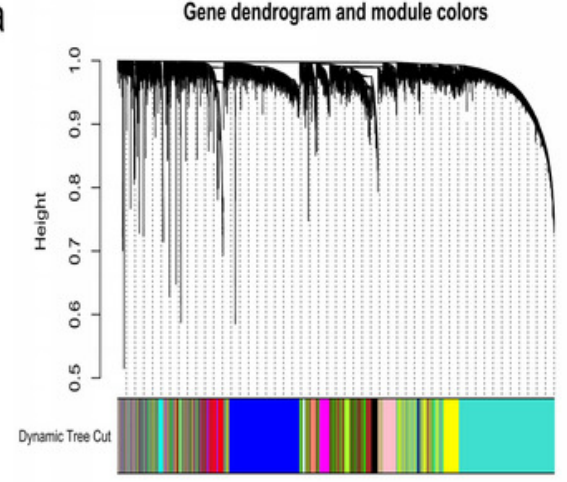

C
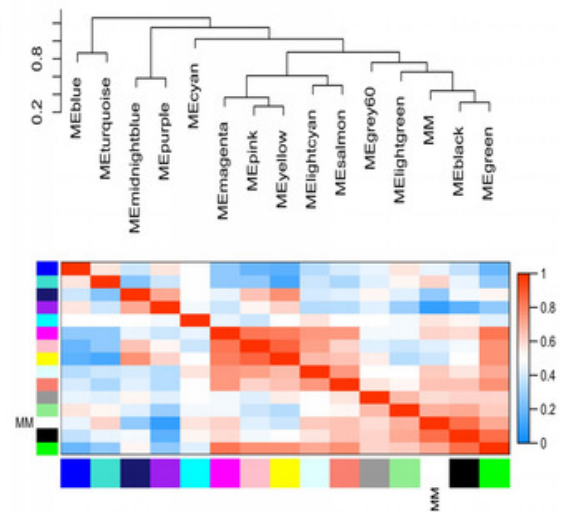

e

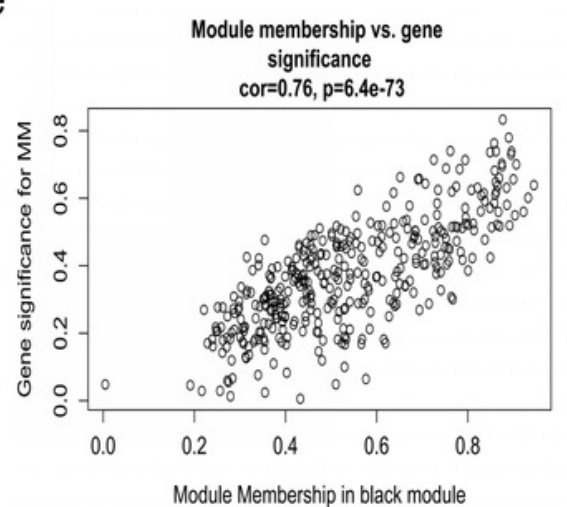

b

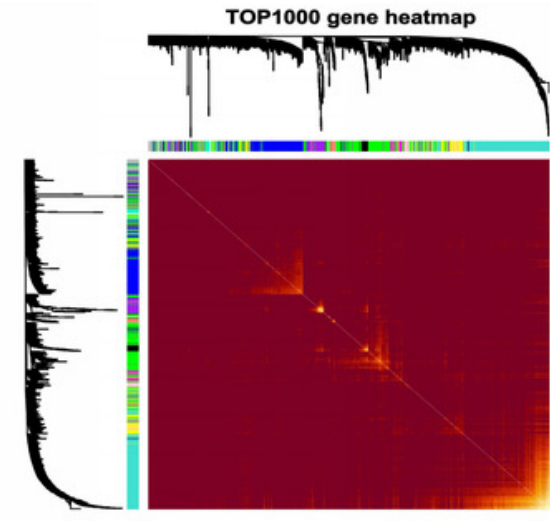

d

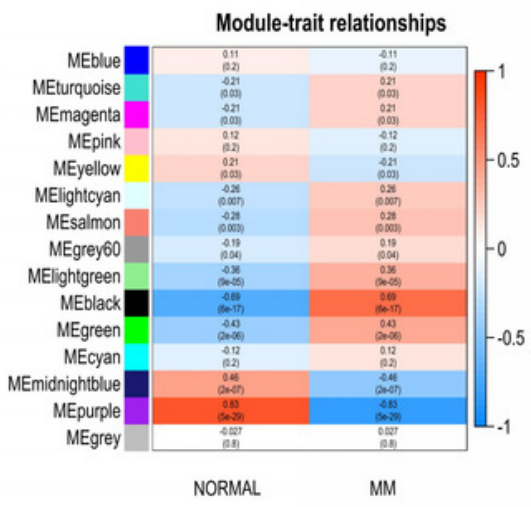

f

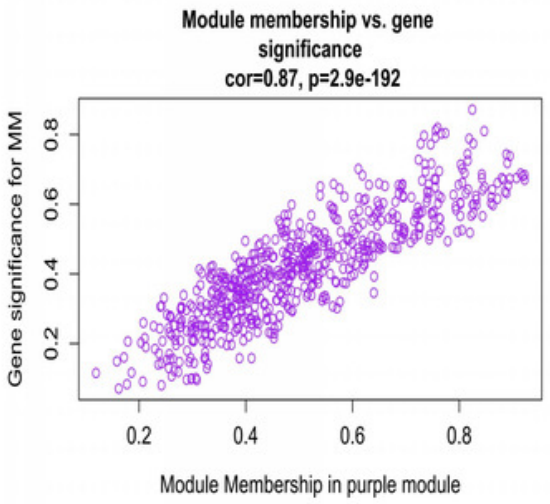


Figure 5

Key genes identified from both the DEGs and the hub genes.

A Venn diagram of the DEGs and hub genes revealed 47 key genes.

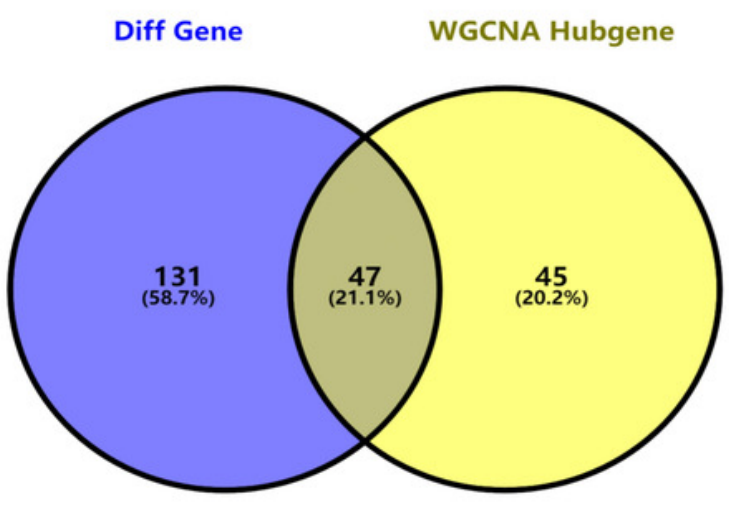


Figure 6

Relationship of the three prognostic genes with OS.

Kaplan-Meier curve showing that the three genes (LYVE1, RNASE1, and RNASE2) expression were associated with OS of MM patients (a-c).

a

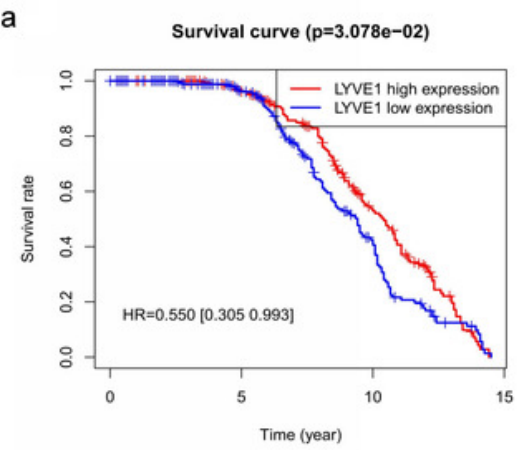

b

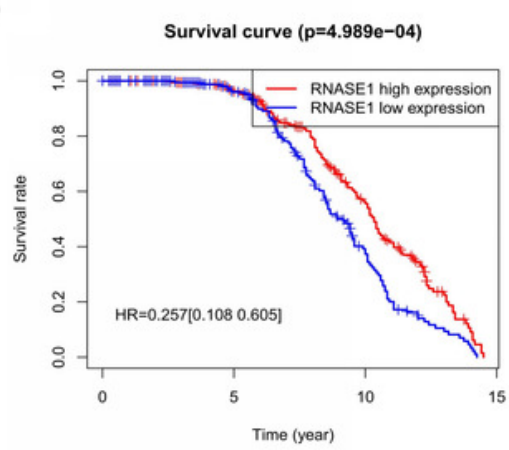

c

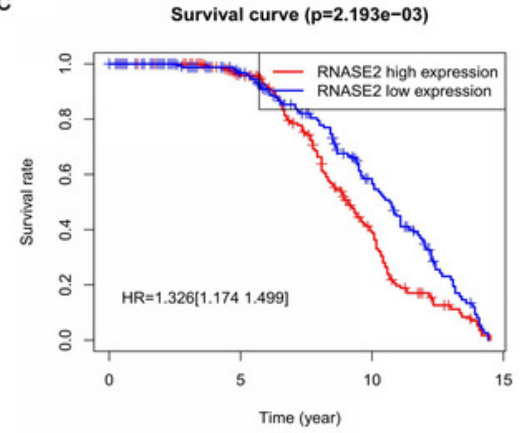


Figure 7

Construction and validation of prognostic risk model for MM patients.

(a-c).Heatmaps of the three prognostic genes in training set, test set, and whole set, respectively. (d-f).Risk score distributions in training set, test set, and whole set, respectively. ( $\mathrm{g}-\mathrm{i})$. Kaplan-Meier curve showing that $\mathrm{OS}$ is significantly shorter for patients in high-risk group than those in low-risk group in training set, test set, and whole set, respectively.

a

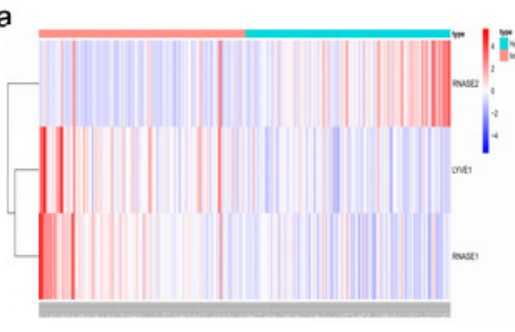

d

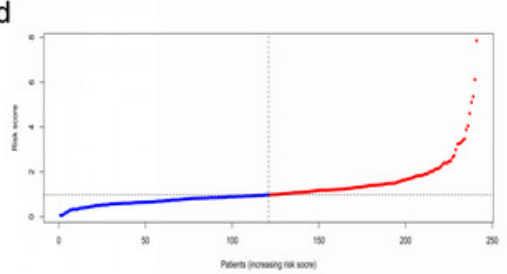

g

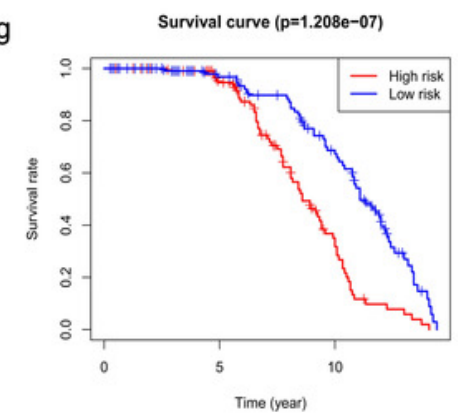

b

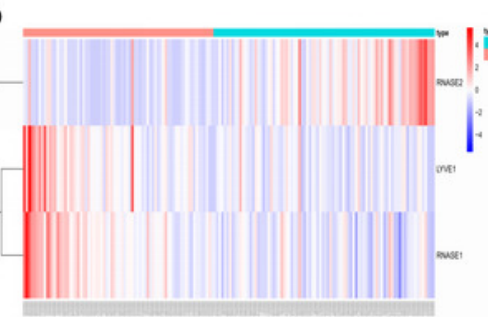

e

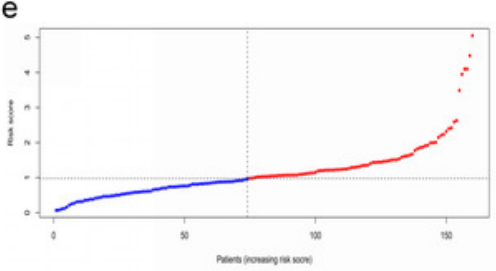

h

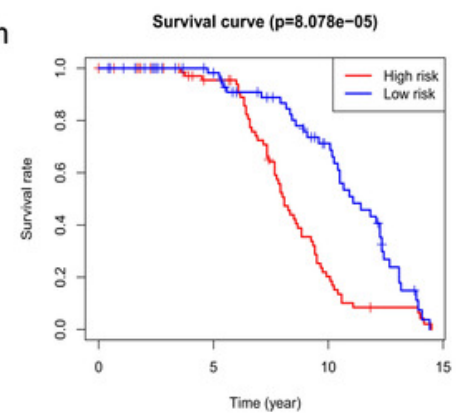

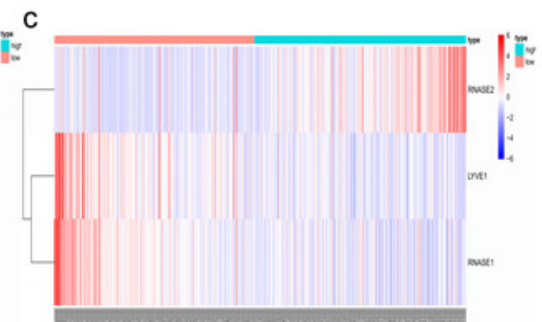

f
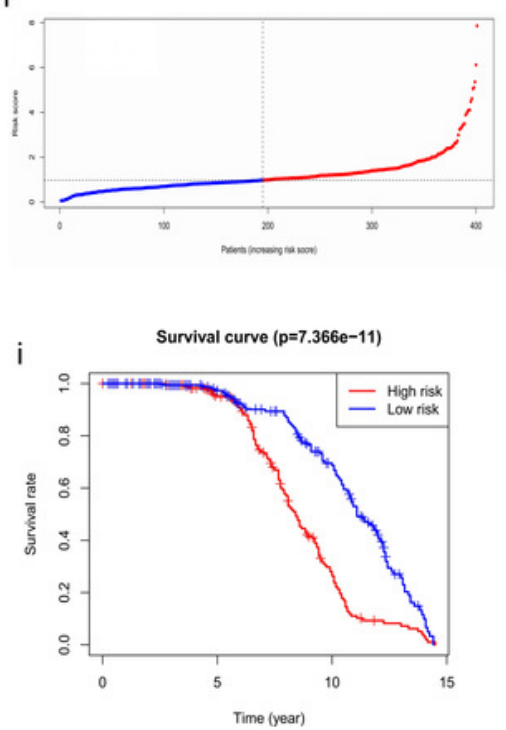
Figure 8

Time-dependent ROC curves for the prognostic model in the GEO MM cohort.

(a-c).Time-dependent ROC curve analysis showing the accuracy and reliability of the prognostic model in training set, test set, and whole set, respectively. (d-f). The ROC curves for 8-, 10-, and 12-year overall survival predictions for the risk model in compare with age, gender, ISS, and R-ISS models in whole set.

a

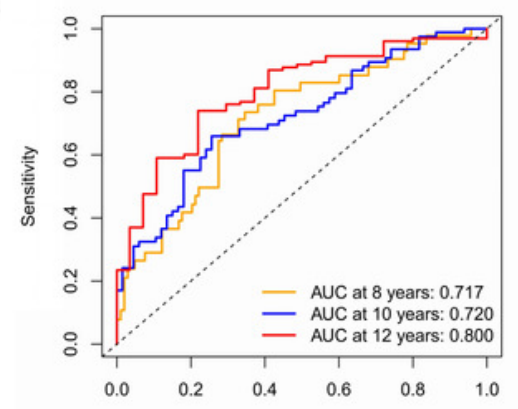

d

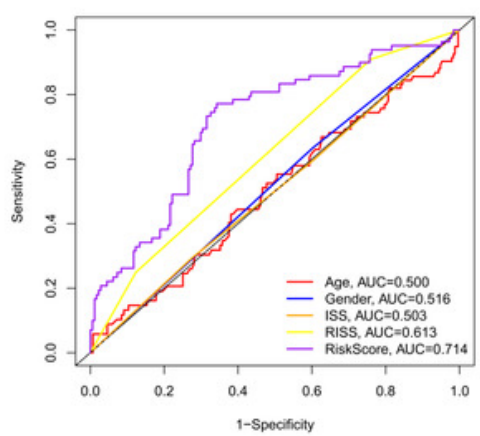

b

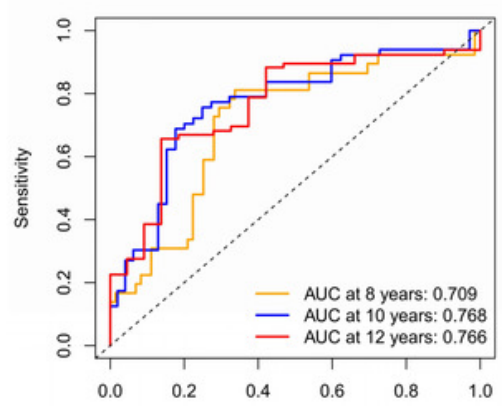

e

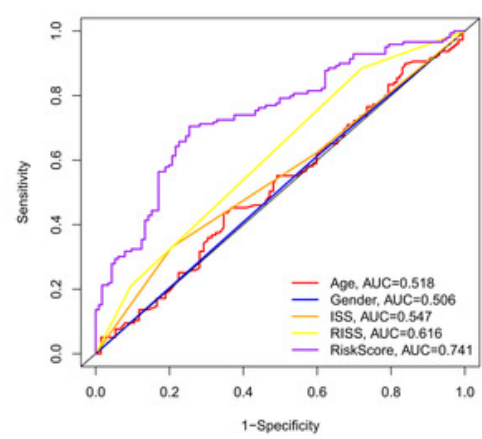

C
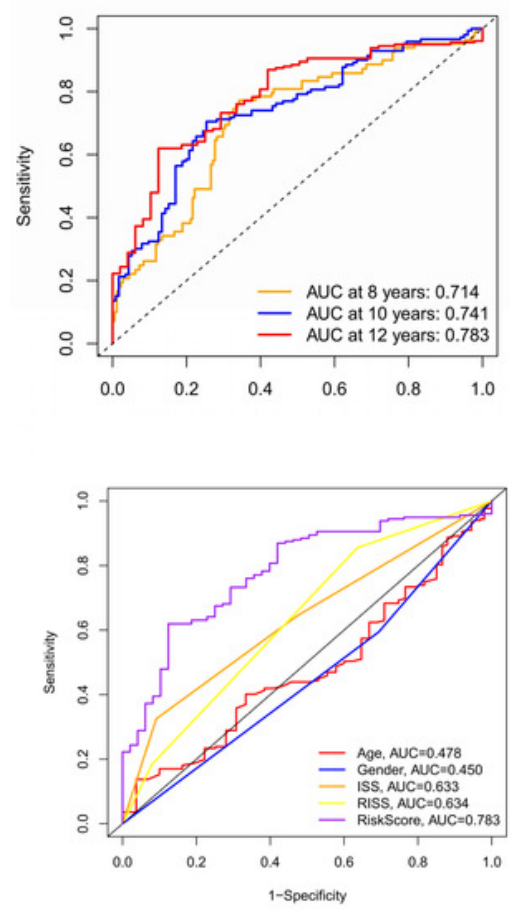
Figure 9

Prognostic risk model could predict prognosis independently.

(a). Univariate association of the prognostic model and clinical characteristics with OS. (b). Multivariate association of the prognostic model and clinical characteristics with OS.
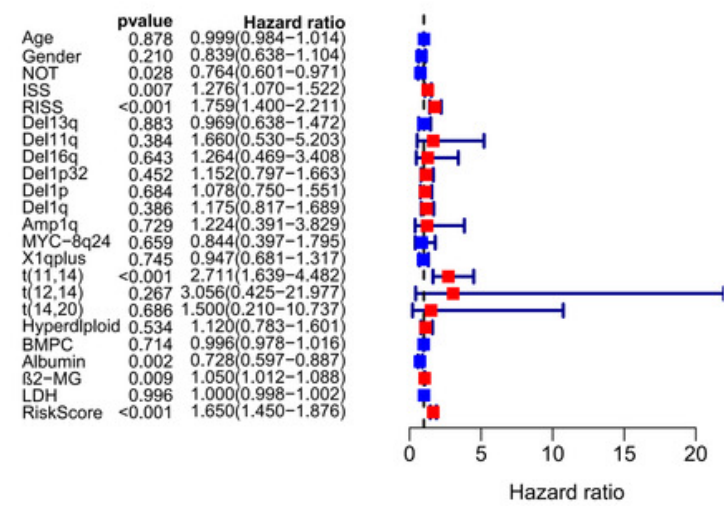

b
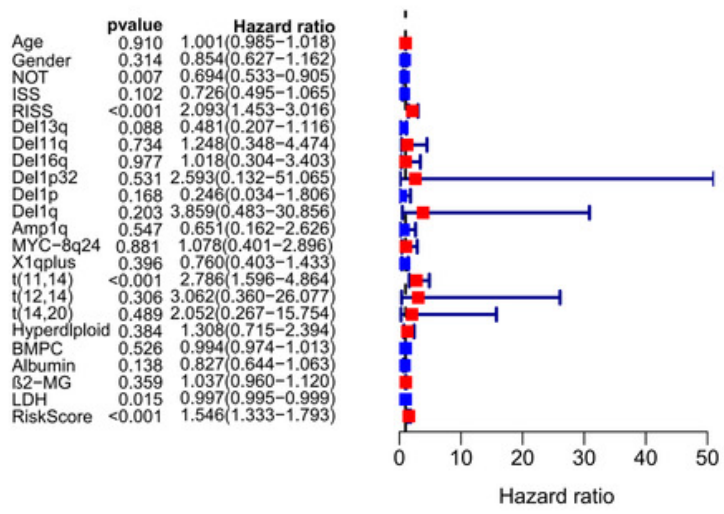
Figure 10

Relationship of prognostic genes and risk model with clinical variables for MM.

(a,b) LYVE1 expression was correlated with ISS and R-ISS stage in MM patients. (c) RNASE1 expression was correlated with R-ISS stage. (d,e) Risk score was significantly correlated with ISS and R-ISS stage.
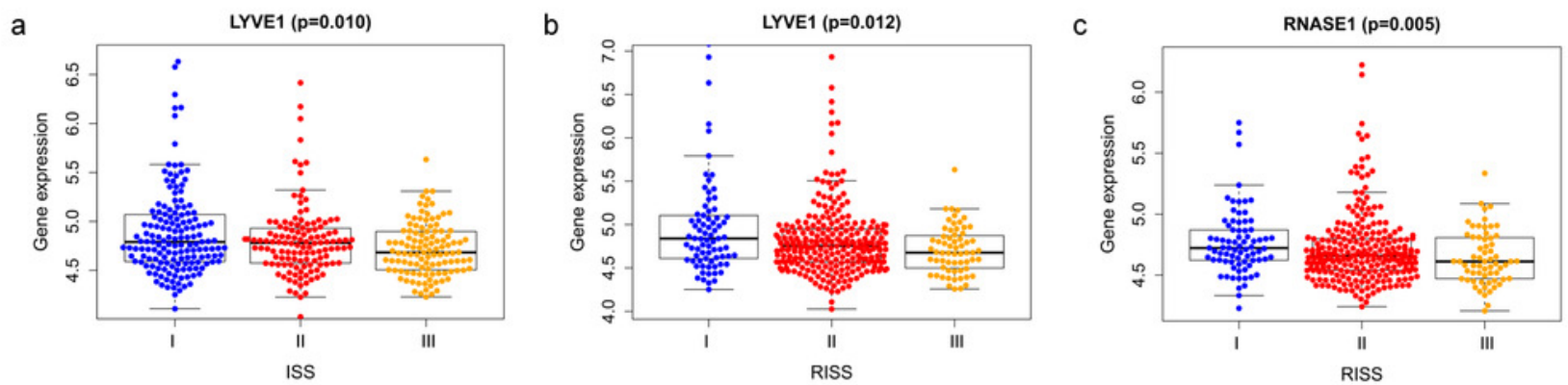

d

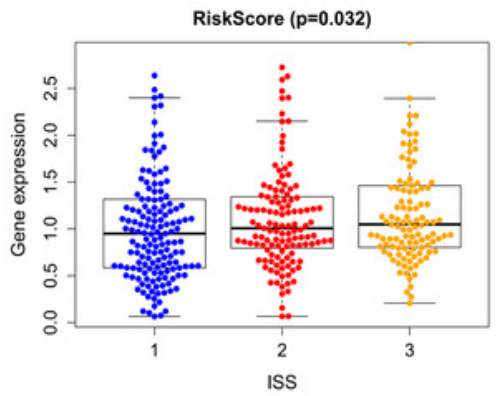

e

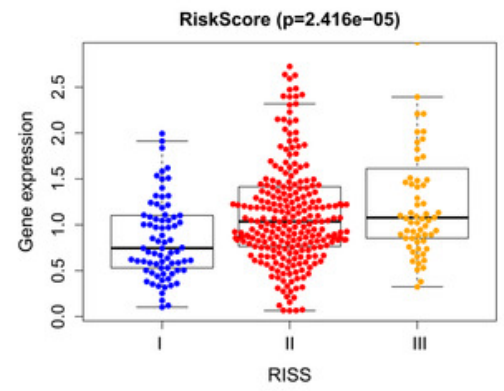




\section{Table $\mathbf{1}$ (on next page)}

Correlation analysis between three genes and clinical characters for MM. 


\begin{tabular}{llllll}
\hline \multirow{2}{*}{ Variables } & $\begin{array}{l}\text { Age }(\leq 60,>60) \\
\mathrm{t}(\mathrm{p})\end{array}$ & $\begin{array}{l}\text { Gender }(\text { female, } \\
\text { male) } \mathrm{t}(\mathrm{p})\end{array}$ & $\begin{array}{l}\mathrm{NTO}(\leq 1,>1) \\
\mathrm{t}(\mathrm{p})\end{array}$ & $\begin{array}{l}\text { ISS (I, II, } \\
\mathrm{III}) \mathrm{t}(\mathrm{p})\end{array}$ & $\begin{array}{l}\text { RISS(I, II, } \\
\mathrm{III}) \mathrm{t}(\mathrm{p})\end{array}$ \\
\hline LYVE1 & 236.601 & $-2.477^{*}$ & 3.895 & $9.293^{*}$ & $8.888^{*}$ \\
RNASE1 & 208.63 & -1.529 & 5.652 & 5.13 & $10.782^{* *}$ \\
RNASE2 & 239.084 & $-2.524^{*}$ & 7.929 & 0.167 & 3.094 \\
Risk score & 238.985 & -0.208 & 5.607 & $6.887^{*}$ & $21.262^{* * *}$ \\
\hline
\end{tabular}

$\mathrm{t}$ : $\mathrm{t}$ value from Student's $\mathrm{t}$ test; $\mathrm{p}$ : $\mathrm{p}$-value from Student's test ${ }^{*} \mathrm{p}<0.05 ;{ }^{* *} \mathrm{p}<0.01 ; * * * \mathrm{p}<0.001$. 
